\title{
FACTORES DEL ANIMAL Y EL MANEJO PREDESTETE QUE AFECTAN LA EDAD AL PRIMER PARTO EN HATOS DE LECHERÍA ESPECIALIZADA DE COSTA RICA ${ }^{1}$
}

\author{
Gloriana Castillo-Badilla ${ }^{2 *}$, Bernardo Vargas-Leitón ${ }^{* *}$, Frank Hueckmann-Voss ${ }^{* * *}$, \\ Juan José Romero-Zúñiga*****
}

\begin{abstract}
Palabras clave: Variables modificables; variables no modificables; sistemas de crianza; desempeño reproductivo; lechería especializada.

Keywords: Modifiable variables; non-modifiable variables; rearing systems; reproductive performance; specialized dairy.
\end{abstract}

Recibido: $14 / 06 / 18$

RESUMEN

La edad al primer parto (EPP) corresponde a uno de los parámetros más importantes para medir la eficiencia productiva de los bovinos, y a su vez, influye en el rendimiento reproductivo y la composición de la leche. El objetivo de este trabajo fue determinar el efecto de los factores del animal y del ambiente, modificables y no modificables, desde el nacimiento hasta el destete sobre la edad al primer parto (EPP) en hatos lecheros especializados de Costa Rica. Se realizó un estudio cohorte retrospectivo para cuantificar el efecto de estas variables sobre la edad al primer parto (EPP), en el estudio se incluyeron, un total de 2980 vacas de razas Jersey y Holstein que presentaron su primer parto entre el 2013 y 2015 , pertenecientes a 22 hatos lecheros especializados, ubicados en diferentes zonas ecológicas de Costa Rica, con datos en el programa VAMPP Bovino. Se analizaron

1 Este trabajo formó parte de la tesis de maestría en Producción Animal Sostenible de la primera autora.

2 Autora para correspondencia. Correo electrónico: gloriana.castillo.badilla@una.cr

Universidad Nacional de Costa Rica, Escuela de Medicina Veterinaria, Cátedra de Salud de Hato y Control de la Producción, Heredia, Costa Rica. (iD) 0000-0002-9005-2423.
Aceptado: 27/08/18

\begin{abstract}
Animal effect and preweanig management on age at first calving in specialized dairy farms of Costa Rica. The age at first calving (AFC) correspond to one of the most important parameters to quantify the productive efficiency of bovines, because it influences the productive performance and milk composition. The aim of the study was to quantify the effect of the animal and environmental variables, modifiable and nonmodifiable, from birth to weaning, on AFC in specialized dairy herds in Costa Rica. A cohort retrospective study was conducted, including a total of 2980 Jersey and Holstein cows with AFC in the period between 2013 and 2015, belonging to 22 specialized dairy herds located in different ecological zones of Costa Rica, with data in the Bovine VAMPP program. All data in the present research, was analyzed by descriptive analysis
\end{abstract}

\footnotetext{
** Universidad Nacional de Costa Rica, Posgrado Regional en Ciencias Veterinarias Tropicales, Heredia, Costa Rica. (D) 0000-0002-1778-9672.

*** Consultor independiente, Heredia, Costa Rica. (iD) 0000-0001-9674-5469.

**** Universidad Nacional de Costa Rica, Posgrado Regional en Ciencias Veterinarias Tropicales, Heredia, Costa Rica. (D) 0000-0002-5252-1604.
} 
los datos mediante estadística descriptiva y un modelo lineal generalizado. La EPP promedio, para los animales en estudio, es 27,4 meses $( \pm 4,7)$. Variables no modificables del individuo, como la raza, presentaron efectos significativos en la EPP, con valores de 30,1 (IC95\%: 29,8-30,4) y 28,4 meses (IC95\%: 28,1-28,8; p<0,001) en la raza Holstein y Jersey, respectivamente. Por otra parte, se determinó un efecto significativo de la variable modificable de manejo ( $\mathrm{p}<0,0001)$, en aquellas fincas con protocolos de alta calidad de crianza de terneras, se obtuvo una EPP de 27,6 meses (IC95\%: 27,2-28,0), significativamente menor a la encontrada en las fincas con desarrollo pobre de reemplazos (EPP: 30,2; IC95\%: 29,8-30,6). Esta tendencia se mantuvo, tanto en el análisis de la variable de manejo de forma independiente como en asociación con raza y finca. La eficiencia de las técnicas de crianza se reflejaron en mejores valores de EPP.

\section{INTRODUCCIÓN}

La edad al primer parto (EPP) corresponde al tiempo que tarda un animal en alcanzar su madurez y reproducirse por vez primera (Hare et al. 2006). El promedio para la EPP, en países de clima templado, oscila entre los 24 y 26 meses (Pirlo et al. 2000). En el caso de Costa Rica la EPP reportada, para animales Jersey (Castillo Badilla et al. 2013) y Holstein (Salazar-Carranza et al. 2013), corresponde a 29,6 ( $\pm 6,8)$ y 30,7 $( \pm 6,8)$ meses, respectivamente. El valor elevado es relacionado con las características climáticas de las zonas de producción de leche, así como a la deficiencia de productos nutricionales de alta calidad (Wing-Ching Jones et al. 2008, SalazarCarranza et al. 2013, Castillo Badilla et al. 2013).

Se ha determinado que la EPP tiene una estrecha relación con variables como el peso, estatura y desarrollo de la novilla (Brand et al. and a generalized linear model. The average AFC for the animals in the present study was 27.4 months $( \pm 4.7)$. Non- modifiable variables, like breed, had significant effect over AFC, with 30,1 (CI95\%: 29.8-30.4) and 28.4 months (CI 95\%: 28.1-28.8) for Holstein and Jersey respectively. Furthermore, the results showed a significant effect of management as a modifiable variable $(\mathrm{p}<0.0001)$ revealing that farms with high quality calf rearing protocols had AFC of 27.63 months (CI95\%: 27.24-28.03), contrary to poor quality systems (AFC: 30.20; CI95\%: 29.83-30.57). This tendency was consistent whether the management variable was included as independent in the model or associated to breed and herd factors, where high efficiency in calf rearing reflects into better values of AFC.

1997, Ettema y Santos 2004). La tasa de crecimiento de una ternera y su peso vivo a la EPP es un punto de referencia sobre el manejo de una finca lechera, asimismo, el desarrollo de una ternera durante sus primeros 90 días de vida define el futuro productivo del animal, ya que es durante los primeros 3 meses de vida que se produce la maduración ruminal y se desarrolla la capacidad de consumo de alimento sólido y forraje (Pithua et al. 2009, Meale et al. 2017). Al respecto, se ha documentado que a menor peso vivo a la EPP, las posibilidades de presentar distocia o tener un menor rendimiento productivo se incrementan (Roche et al.2015) por lo cual el balance entre los 3 factores es fundamental para obtener el máximo potencial de la hembra en el hato.

Uno de los principales problemas en las explotaciones bovinas es la mortalidad de los animales jóvenes, en el Reino Unido entre 13-16\% de las terneras no llegan a su primer parto (Brickell 
y Wathes 2011). Por ese motivo, el tipo de manejo de una ternera, durante sus primeros meses de vida, como el tipo de alojamiento, las prácticas de salud y el tipo de alimentación, son críticos para evitar la ocurrencia y gravedad de enfermedades, que a su vez pueden provocar incrementos de la EPP (Lievaart et al. 2012).

En Costa Rica se han conducido varios estudios sobre factores que afectan la EPP y, a su vez, el efecto de esta sobre parámetros productivos y reproductivos, especialmente en la primera lactancia y sobre su vida productiva (VargasLeitón y Ulloa 2008, Castillo Badilla et al. 2013, Salazar-Carranza et al. 2013). Sin embargo, hasta la fecha, no existen estudios, a nivel poblacional, que relacionen variables de manejo durante período de crianza con factores de la madre, el individuo y el ambiente.

Las variables que pueden afectar la EPP, pueden llegar a ser modificadas de forma inmediata por el productor, lo cual hace posible un cambio en la estrategia de crianza de una finca en particular, para mejorar su rendimiento productivo y reproductivo. En los últimos años, se ha involucrado el término de variables modificables y no modificables en estudios de epidemiología clínica humana y en producción animal, para identificar elementos que pueden controlarse, modificarse o tratarse por el ser humano para obtener un beneficio en particular (Ibekwe 2015, Mee et al. 2014). Las variables no modificables corresponden a atributos del individuo, que no pueden ser alterados o ajustados, por ejemplo: la edad, raza, genética, entre otros (Ibekwe 2015).

Los primeros 3 meses de vida son los de mayor vulnerabilidad de los animales de producción a cambios de manejo; además, el manejo de las hembras en las fincas especializadas de Costa Rica, luego del destete, es bastante homogéneo, pues los animales se mantienen en pastoreo extensivo. Así, este estudio busca evaluar factores modificables en la etapa de crianza de las terneras, desde el nacimiento hasta el destete a los 90 días, tales como las prácticas de salud, alojamiento y alimentación; y no modificables, como la raza, factores de la madre, sobre la edad al primer parto en hatos lecheros especializados de Costa Rica.

\section{MATERIALES Y MÉTODOS}

\section{Población y diseño del estudio}

El estudio se realiza en 22 fincas de las principales zonas lecheras de Costa Rica: 11 fincas de la Región Central: Coronado (1), Cartago ( $\mathrm{n}=5)$, Poás $(n=1)$, Vara Blanca $(n=3)$ y Los Cartagos $(\mathrm{n}=1)$; 9 de la región Huetar Norte: Ciudad Quesada $(n=3)$, Sucre $(n=1)$, Aguas Zarcas ( $n=3)$ y Río Cuarto ( $\mathrm{n}=2) ; 1$ en la región Chorotega (Guayabo) y 1 en Pacífico Central (Caldera) (Cuadro 1). 
Cuadro 1. Características de las explotaciones estudiadas.

\begin{tabular}{|c|c|c|c|c|c|c|c|c|c|}
\hline Finca & $\begin{array}{c}\text { Raza } \\
\text { Predominante }\end{array}$ & Estabulado & $\begin{array}{l}\text { Número } \\
\text { de } \\
\text { Animales }\end{array}$ & $\begin{array}{l}\text { Producción } \\
\text { promedio } \\
\text { a } 305 \text { días }\end{array}$ & EPP & SPC & Alojamiento & $\begin{array}{c}\text { Técnica de } \\
\text { Alimentación }\end{array}$ & $\begin{array}{c}\text { Tipo } \\
\text { de } \\
\text { leche }\end{array}$ \\
\hline & & & & \multicolumn{3}{|c|}{ VACAS } & \multicolumn{3}{|c|}{ TERNERAS } \\
\hline 1 & Jersey & $\mathrm{E}$ & 638 & 5958,46 & 23,72 & 1,74 & $\mathrm{Si}$ & Ch-B & $\mathrm{R}$ \\
\hline 2 & Jersey & $\mathrm{P}$ & 71 & 6060,33 & 27,80 & 1,91 & $\mathrm{Ei}$ & $\mathrm{Ch}-\mathrm{B}$ & $\mathrm{L}$ \\
\hline 3 & Holstein & S & 158 & 7678,52 & 24,06 & 1,97 & $\mathrm{Eg}$ & Asa & $\mathrm{L}$ \\
\hline 4 & Jersey & $\mathrm{P}$ & 75 & 2773,41 & 25,50 & 1,51 & $\mathrm{Ei}$ & Ch-B & $\mathrm{L}$ \\
\hline 5 & Jersey & S & 66 & 4843,42 & 25,65 & 2,05 & $\mathrm{Ei}$ & Ch-B & $\mathrm{L}$ \\
\hline 6 & Holstein & $S$ & 140 & 5228,63 & 28,33 & 1,74 & $\mathrm{Ei}$ & $\mathrm{Ch}$ & $\mathrm{R}$ \\
\hline 7 & Jersey & S & 330 & 6267,24 & 24,23 & 1,90 & $\mathrm{Ei}$ & Ch-B & L-R \\
\hline 8 & Holstein & S & 400 & 6096,79 & 26,87 & 2,14 & $\mathrm{Ei}$ & Ch-B & $\mathrm{L}$ \\
\hline 9 & Jersey & $\mathrm{P}$ & 300 & 6130,58 & 24,79 & 1,97 & $\mathrm{Ei}$ & Ch-B & $\mathrm{L}$ \\
\hline 10 & Holstein & $\mathrm{P}$ & 71 & 4411,40 & 28,77 & 1,59 & $\mathrm{Ei}$ & $\mathrm{Ch}-\mathrm{B}$ & $\mathrm{R}$ \\
\hline 11 & Jersey & $\mathrm{P}$ & 256 & 4382,42 & 30,55 & 1,77 & $\mathrm{Eg}$ & B & $\mathrm{L}$ \\
\hline 12 & Jersey & S & 124 & 4742,03 & 26,00 & 1,39 & $\mathrm{Ei}$ & B & $\mathrm{L}$ \\
\hline 13 & Holstein & $S$ & 390 & 3355,74 & 30,79 & 2,90 & $\mathrm{Ei}$ & B & $\mathrm{L}$ \\
\hline 14 & Holstein & $S$ & 46 & 3061,38 & 34,88 & 2,80 & $\mathrm{Ei}$ & Ch-B & $\mathrm{R}$ \\
\hline 15 & Holstein & $\mathrm{P}$ & 170 & 5244,33 & 30,82 & 1,83 & $\mathrm{Ei}$ & B & $\mathrm{L}$ \\
\hline 16 & Jersey & $S$ & 564 & 4126,56 & 23,61 & 1,69 & $\mathrm{Ei}$ & Ch-B & $\mathrm{L}$ \\
\hline 17 & Holstein & S & 761 & 6343,12 & 30,30 & 1,79 & $\mathrm{Sg}$ & B & $\mathrm{R}$ \\
\hline 18 & Holstein & $\mathrm{P}$ & 650 & 3986,36 & 33,29 & 1,17 & $\mathrm{Ei}$ & B & $\mathrm{L}$ \\
\hline 19 & Jersey & $\mathrm{P}$ & 120 & 5395,65 & 31,58 & 2,04 & $\mathrm{Ei}$ & B & $\mathrm{L}$ \\
\hline 20 & Holstein & $\mathrm{S}$ & 73 & 6498,83 & 26,16 & 1,95 & $\mathrm{Sg}$ & Asa & $\mathrm{L}$ \\
\hline 21 & Jersey & S & 704 & 7432,72 & 24,29 & 1,72 & $\mathrm{Ei}$ & Ch-B & $\mathrm{L}$ \\
\hline 22 & Jersey & $\mathrm{P}$ & 15 & 4356,90 & 28,96 & 2,22 & $\mathrm{Eg}$ & $\mathrm{Ch}$ & $\mathrm{L}$ \\
\hline
\end{tabular}

Estabulado: E (estabulado) S (Semiestabulado) P (Pastoreo); EPP: Edad al Primer Parto; SPC: Servicios por concepción; Alojamiento: Ei (Estabulado individual), Eg (Estabulado grupal), Si (Semiestabulado-individual), Sg (Semiestabulado-grupal); Técnica de Alimentación: Ch-B (chupón y Balde), Ch (chupón), B (Balde), Asa (Alimentador semiautomático); Tipo de leche: L (leche entera), R (Reemplazador de leche), L-R (Reemplazador y leche entera).

Se utiliza un diseño de estudio cohorte retrospectivo, con datos provenientes de hembras registradas en el programa VAMPP Bovino 3,0 (Noordhuizen y Buurman 1984) de hatos lecheros especializados de Costa Rica, que presentan su primer parto entre el 2013 y el 2015, con registros completos para todas las variables del estudio. 


\section{Recolección de datos y descripción de las variables}

Se utilizó la base nacional de datos de las fincas registradas en el programa VAMPP Bovino 3,0, en el Centro Regional de Informática para la Producción Animal Sostenible (CRIPAS), del Programa de Investigación en Medicina Poblacional (MedPob), de la Escuela de Medicina Veterinaria-Universidad Nacional (EMV-UNA) (Pérez et al. 1989, Dwinger et al. 1994, Baaijen y Pérez 1995).

De forma complementaria, se realizó una encuesta a los responsables de los hatos, para conocer las prácticas de manejo relacionadas con la crianza de los reemplazos durante el periodo que comprende desde el nacimiento hasta el destete, que se realiza en cada lechería, en el que se tomo en consideración que la edad al destete es de 90 días. Se tomó en cuenta que estas ganaderías dispusieran de información completa respecto a registros productivos, reproductivos, sanitarios y alimenticios; que, además, se contara con la anuencia del propietario para facilitar la información complementaria: protocolos de vacunación, desparasitación, limpieza, pastos, entre otros.

La variable dependiente corresponde a la edad a primer parto (EPP, en meses) y las variables independientes se clasifican como asociadas al animal o ambiente. Dentro de las variables de animal incluidas se encuentran la raza (Jersey o Holstein), el año y mes de nacimiento (según calendario), servicios por concepción efectivos (1 SPCE, de >1-2 SPCE, >2 SPCE), y el intervalo primer celo-concepción $(<31$ días IPCC, 31-60 IPCC, 61-90 IPCC, $\geq 91$ días IPCC).

La clasificación de modificables o no modificables se otorga según la posibilidad de ser cambiadas por el responsable del hato, por ejemplo: el manejo nutricional se puede cambiar al incorporar nuevos ingredientes, o técnicas de alimentación. Por el contrario, variables, como la raza, no son alterables (razonablemente) por actividades de manejo.

A partir de la información de la encuesta, se generaron 39 variables, las cuales se califican en 3 áreas: salud, alojamiento o alimentación
(Castillo-Badilla et al. 2019). En el área de salud se incluyen aquellas variables relacionadas con el estatus sanitario del hato, tales como: protocolos de vacunación, políticas de reemplazos, higiene, visita regular de un médico veterinario, prevalencia de enfermedades gastrointestinales, respiratorias y onfaloflebitis, registro de muertes pre-destete y manejo de los casos clínicos. En el área de alojamiento, se consideró el tipo de estabulado; si se alojaban de forma grupal o individual; el tipo de piso, si este era o no elevado; el tipo de cama y la división entre una cuna y otra. Para área de alimentación se incluyeron variables como: el tipo de leche ofrecida a la ternera ya sea reemplazador o leche entera; si esta se sometía a algún tipo de análisis determinado, como medición de células somáticas; ausencia de mastitis o residuos de antibiótico; si se pasteurizaba, previo a ofrecerla a las terneras; igualmente, si la dieta de las terneras tenía aporte de concentrado y heno o pasto fresco.

Seguidamente, se asignaron puntuaciones a cada variable dentro de cada categoría por medio de escalas discretas ponderadas de acuerdo con la importancia relativa de cada variable. De acuerdo con la escala utilizada, la máxima puntuación posible, para una finca con condiciones óptimas en todas las variables, es de 80 en el área de salud; 15, en el área de alojamiento; 27, en el área de alimentación; para un total de 122 en el manejo global.

Seguidamente, se realizó la sumatoria de los puntajes otorgados para las 3 áreas de manejo: salud, alimentación y alojamiento, para obtener la puntuación de la variable de manejo global. Para poder clasificar las fincas según el tipo de manejo en: alta, media o baja, se calculó el valor de los quintiles, para utilizar los valores de la sumatoria como referencia para la categorización. Por lo tanto, se clasificaron como fincas con manejo alto a aquellas con más de 101 puntos (quintil superior), media con puntuación entre 75 y 101 y baja con menos de 75 puntos (quintil inferior).

\section{Análisis estadístico}

Para el análisis del efecto de las variables de animal y ambiente sobre la EPP, se utilizó 
un Modelo Lineal Generalizado (GLM, por sus siglas en inglés). Esta herramienta de análisis logra combinar las características de los modelos lineales mixtos, los cuales incorporan efectos aleatorios y modelos lineales generalizados, que pueden analizar datos con distribución no normal (Bolker et al. 2009).

El modelo estadístico para explicar la EPP es:

$$
Y=\mu+\beta_{1} M g+\beta_{2} A n+\beta_{3} M n+\beta_{4} R+\beta_{5} S P C E+\beta_{6} I P C C+\beta_{7}(R \times M g)+\beta_{8} H(R \times M g)+\xi
$$

En el cual:

$Y=$ variable dependiente (EPP en meses).

$\mu=$ media general.

$\beta_{1} \mathrm{Mg}=$ efecto fijo de grupo de manejo (Alto, medio o bajo, según sumatoria global).

$\beta_{2} A n=$ efecto fijo de año de nacimiento.

$\beta_{3} \mathrm{Mn}=$ efecto fijo de mes de nacimiento.

$\beta_{4} R=$ efecto fijo de raza.

$\beta_{5} \mathrm{SPCE}=$ efecto de los servicios por concepción efectivos (en categorías: 1,2, $\geq 3$ ).

$\beta_{6} I P C C=$ efecto fijo del intervalo primer celoconcepción (en categorías: 0: <30 días, 1: 30 a 60 días, 2: 61 a 90 días, 3: $\geq 91$ días).

$\beta_{7}\left(R^{*} M g\right)=$ efecto fijo de la interacción raza por grupo de manejo.

$\beta_{8} H\left(R^{*} M g\right)=$ efecto fijo del hato anidado dentro de grupos de raza y manejo.

$\xi=$ error residual aleatorio.

Los análisis estadísticos se realizaron con el programa SAS, versión 9,4, con su rutina GLIMMIX (SAS Institute Inc 2010). En el modelo, se asumió una distribución lognormal para la variable de respuesta (EPP); se utilizó una función de enlace identidad.

\section{RESULTADOS Y DISCUSIÓN}

Se analizaron un total de 2980 primerizas, pertenecientes a 22 fincas distribuidas en diferentes zonas ecológicas de Costa Rica. La EPP promedio, de la totalidad de vacas incluidas, fue de 27,4 meses $( \pm 4,7)$. Los indicadores de reproducción, registraron un promedio de $1,8( \pm 1,3)$ servicios por concepción efectivos (SPCE) y 44,7 días $( \pm 57,6)$ de intervalo primer celo concepción (IPCC).

El modelo evaluado convergió satisfactoriamente $(\mathrm{p}<0,001)$ con un cumplimiento adecuado de los supuestos de normalidad, independencia y varianza constante de los residuos. Asimismo, se presentó un efecto significativo de la mayoría de las variables de animal y ambiente sobre la EPP (Cuadro 2). 
Cuadro 2. Medias marginales (e intervalos de confianza 95\%) de Edad a Primer Parto (EPP, días) para los distintos estratos de los factores relacionados al animal y ambiente.

\begin{tabular}{|c|c|c|c|c|c|}
\hline \multirow{2}{*}{ Factor } & \multirow{2}{*}{ Estrato } & \multirow{2}{*}{$\begin{array}{c}\text { EPP } \\
\text { Estimado }\end{array}$} & \multicolumn{2}{|c|}{ Intervalo de Confianza 95\% } & \multirow{2}{*}{ Valor de $\mathrm{P}$} \\
\hline & & & LI & LS & \\
\hline Intercepto & & 34,1 & & & $<0,0001$ \\
\hline \multirow[t]{2}{*}{ Raza } & Holstein & 30,1 & 29,8 & 30,4 & $<0,0001$ \\
\hline & Jersey & 28,4 & 28,1 & 28,8 & \\
\hline \multirow[t]{5}{*}{ Año de nacimiento } & 2010 & 31,8 & 31,4 & 32,2 & $<0,0001$ \\
\hline & 2011 & 28,9 & 28,7 & 29,2 & \\
\hline & 2012 & 29,0 & 28,7 & 29,3 & \\
\hline & 2013 & 28,8 & 28,5 & 29,0 & \\
\hline & 2014 & 28,0 & 27,5 & 28,5 & \\
\hline \multirow[t]{12}{*}{ Mes de nacimiento } & Enero & 29,7 & 29,4 & 30,1 & 0,0012 \\
\hline & Febrero & 29,7 & 29,3 & 30,0 & \\
\hline & Marzo & 29,5 & 29,1 & 29,8 & \\
\hline & Abril & 29,3 & 28,9 & 29,7 & \\
\hline & Mayo & 29,4 & 29,0 & 29,8 & \\
\hline & Junio & 29,2 & 28,8 & 29,6 & \\
\hline & Julio & 29,2 & 28,8 & 29,6 & \\
\hline & Agosto & 29,3 & 29,0 & 29,7 & \\
\hline & Setiembre & 28,8 & 28,4 & 29,2 & \\
\hline & Octubre & 29,2 & 28,8 & 29,6 & \\
\hline & Noviembre & 29,1 & 28,8 & 29,5 & \\
\hline & Diciembre & 28,9 & 28,5 & 29,3 & \\
\hline \multirow[t]{3}{*}{ SPCE } & 1 & 27,8 & 27,5 & 28,1 & $<0,0001$ \\
\hline & 2 & 29,3 & 29,0 & 29,6 & \\
\hline & 3 & 30,8 & 30,4 & 31,2 & \\
\hline \multirow[t]{4}{*}{ IPCC } & 0 & 28,6 & 28,3 & 28,9 & $<0,0001$ \\
\hline & 1 & 28,2 & 27,8 & 28,6 & \\
\hline & 2 & 29,1 & 28,6 & 29,5 & \\
\hline & 3 & 31,3 & 30,9 & 31,8 & \\
\hline \multirow{3}{*}{$\begin{array}{l}\text { Prácticas } \\
\text { manejo }\end{array}$} & Alto & 27,6 & 27,2 & 28,0 & $<0,0001$ \\
\hline & Medio & 30,1 & 29,7 & 30,4 & \\
\hline & Bajo & 30,2 & 29,8 & 30,6 & \\
\hline
\end{tabular}

$* \mathrm{P}=$ Valor de $\mathrm{P}$ para el efecto del factor correspondiente. $\mathrm{LI}=$ límite inferior. $\mathrm{LS}=$ límite superior. $\mathrm{SPCE}=$ servicios por concepción efectivos. IPCC= Intervalo primer celo-concepción (en categorías: 0: <30 días, 1: 30 a 60 días, 2: 61 a 90 días, 3 : $\geq 91$ días). 
Las primerizas Holstein presentaron un valor de EPP de 30,1 (IC95\%: 29,8-30,4) meses; 1,7 meses más que las Jersey (EPP: 28,4; IC95\%: 28,1-28,8; $\mathrm{p}<0,001)$. Estos valores son similares a los reportados por otros estudios en Costa Rica, tales como 30,7 meses ( $\pm 6,8$; IC95\%: 30,6 - 30,8) para la raza Holstein (Salazar-Carranza et al. 2013) y 29,3 ( $\pm 6,8$; IC 95\%: 29,3-29,4) para los animales de raza Jersey (Castillo Badilla et al. 2013). Se hizo evidente la precocidad reproductiva que caracteriza a la raza Jersey, asimismo la capacidad de adaptación climática que esta posee a diferencia de la raza Holstein, lo cual permite alcanzar su madurez sexual a más temprana edad (Washburn et al. 2002).

Se observó que no hubo variación significativa de la EPP entre los años de nacimiento 2010, 2012 y 2013; sin embargo, hubo una diferencia de aproximadamente 4 meses en la EPP, desde el 2010 (EPP: 31,8; IC95\%: 31,4-32,2) al 2014, en el que se reporta una EPP de 28,0 (IC95\%: 27,5-28,5) (Cuadro 1). La tendencia a la disminución se ajustó, a la búsqueda de los productores, a nivel mundial, de obtener valores de EPP entre los 21 y 24 meses de vida, para garantizar una mayor longevidad dentro del hato, pues existe una correlación negativa entre la EPP y la vida productiva (Nilforooshan y Edriss 2004). Además, Hultgren et al. (2011), reportaron que para EPP entre 24 y 31 meses, los animales presentan una vida productiva constante y un mayor retorno económico para el productor; mientras que, por encima de los 31 meses, se observa una disminución de la producción de leche; lo cual, como consecuencia, amenaza la longevidad de la vaca dentro del hato (Ettema y Santos 2004, Changhee Do et al. 2013).

Se determinó, además, que el mes de nacimiento de la hembra afecta significativamente la EPP $(\mathrm{P}=0,0012)$. Los animales nacidos en enero, febrero y marzo presentan los valores más altos de EPP, con 29,7 (IC95\%: 29,4-30,1), 29,6 (IC95\%: 29,3-30,0), у 29,5 (IC95\%: 29,029,8), respectivamente. Por el contrario, las nacidas en setiembre y diciembre tienen las EPP más bajas, con 28,8 (IC95\%: 28,4-29,2) y 28,9
(IC95\%: 28,5-29,2) (Cuadro 1). Este resultado concuerda con los estudios de Castillo Badilla et al. (2013) y Salazar Carranza et al. (2013), quienes encontraron que los animales nacidos en los meses más secos presentan mayores valores de EPP que aquellos nacidos en los meses más lluviosos.

Los efectos provenientes del mes de nacimiento, sobre la edad al primer parto, pueden derivarse, al menos en parte de las diferencias de alimentación en las épocas de verano e invierno, las cuales a su vez, se reflejan en el crecimiento y reproducción de las novillas (Ossa et al. 2007). La EPP está influenciada de manera directa por el peso, tamaño y madurez del animal, factores que bajo condiciones tropicales son difíciles de controlar debido a la influencia del estrés calórico sobre el individuo, el uso de forrajes de baja calidad y la poca o nula suplementación (WingChing Jones et al. 2008).

Los parámetros reproductivos, servicios por concepción efectivos (SPCE) y el intervalo primer celo-concepción (IPCC) presentaron un efecto significativo sobre EPP $(p<0,001)$. Las hembras con mayor cantidad de SPCE mostraron una EPP de 30,8 meses (IC95\%: 30,4-31,2), mientras que las novillas, con un solo servicio, un valor de EPP de 27,8 (IC95\%: 27,5-28,1). Además, las novillas con un IPCC superior a los 91 días revelan los valores de EPP mayores (EPP: 30,2, IC95\%: 30,9-31,8), significativamente distinto a los animales con IPCC inferiores a 90 días (Cuadro 1). La situación se puede relacionar con el desarrollo de la hembra previo a la pubertad, en el cual manejos nutricionales deficientes, pueden ocasionar un retraso en el desarrollo durante esta fase, que puede traer alteraciones en los índices reproductivos (Le Cozler et al. 2008, Cooke et al. 2013). La edad al primer servicio no se tomó en cuenta como variable independiente en este estudio. Algunas personas autoras afirman que, conforme se incrementa la edad de la novilla los índices de fertilidad disminuyen, lo que causa un incremento en los SPCE y IPCC (Le Cozler et al. 2008, Cooke et al. 2013) 
Para este estudio en particular, de forma innovadora se fusionaron de manera ponderada las prácticas de salud, alojamiento y alimentación en una variable global, denominada "manejo".

El manejo en su condición de variable modificable se exploró en el modelo estadístico tanto de forma aislada como en asociación con la raza y la finca. Como los efectos de los factores se han estudiado individualmente, el soporte de la literatura es escaso al explicar los resultados obtenidos bajo una clasificación integrada.

La sumatoria ponderada de las variables parciales, se realizó en manejo global de las 22 fincas utilizadas, 4 clasificadas como explotaciones con manejo alto (Media: 105; $\pm 1,4$ ), 14 de manejo medio (Media: 89,0; $\pm 9,0$ ); y 4 de manejo bajo (Media: 62,$5 ; \pm 11,6$ ), con un total de 659, 1793 y 528 animales.

Las principales inconsistencias se encontraron en el área de salud, que correspondió al criterio más difícil de manejar aunque repercute de forma relevante en los parámetros reproductivos, como la EPP. La falla más común corresponde a las prácticas de higiene y el uso productos de mala calidad o una baja frecuencia de limpieza de instalaciones. Asimismo, el número de casos clínicos, durante el período de desarrollo del animal, repercute en la calificación parcial que tengan las fincas por categoría.

Se observó que los animales sometidos a sistemas de manejo de calidad deficiente o baja, presentan una media marginal de EPP de 30,20 meses (IC95\%: 29,8-30,6), similar a lo obtenido por los animales criados en sistemas con prácticas de manejo intermedias (EPP: 30,05; IC95\%: 29,7-30,4) (Figura 1). Por el contrario los animales con programas de desarrollo de terneras de alta calidad, presentaron los valores de EPP más bajos, en comparación con los otros 2 sistemas, con una EPP de 27,6 meses (IC95\%: 27,2-28,0), lo que evidencia la importancia del manejo efectivo durante el periodo de crianza sobre el desempeño posterior de una novilla, en los parámetros reproductivos y productivos (Heinrichs et al. 2005, Le Cozler et al. 2008, Pithua et al. 2009, Heinrichs y Heinrichs 2011, Abeni et al. 2012).

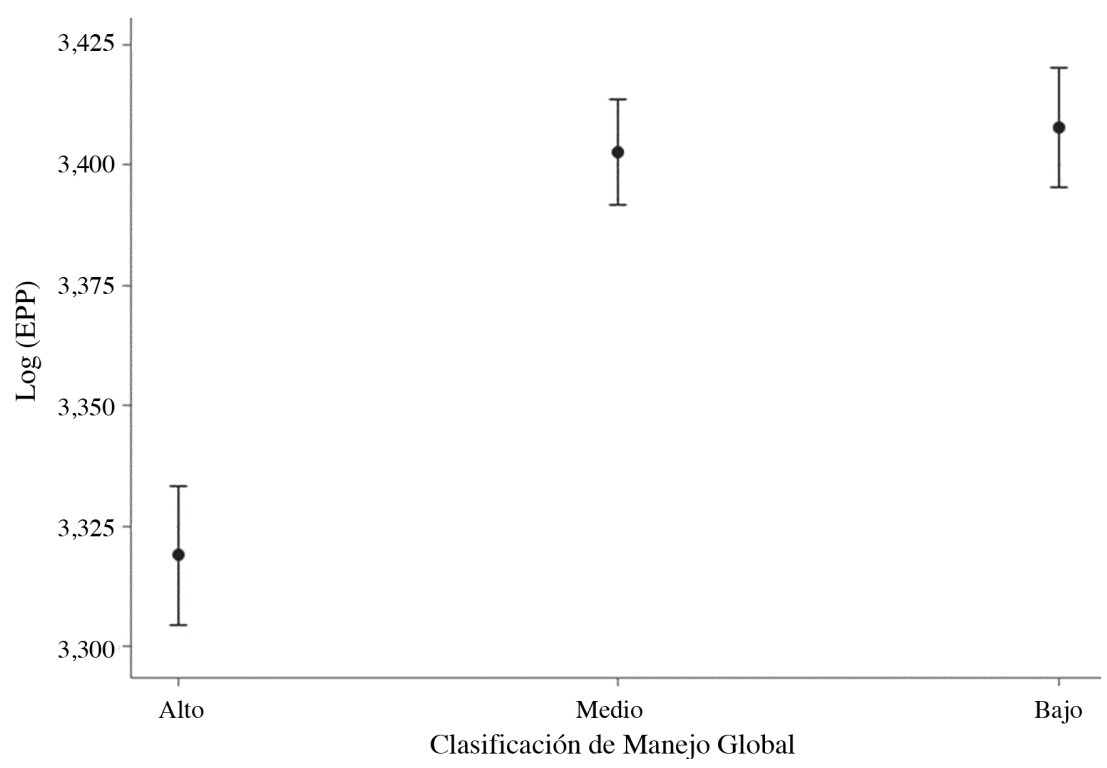

Fig. 1. Medias marginales e intervalo de confianza 95\% para Edad a Primer Parto (escala logarítmica) en grupos de fincas con nivel de manejo Alto, Medio y Bajo. 
La crianza de terneras con un adecuado peso se puede lograr cuando la finca tiene prácticas eficientes de salud, con sus protocolos vacunales completos y atención temprana de casos clínicos, así como alojamiento que cumpla con las características necesarias de confort, higiene y bienestar animal, junto con un balance nutricional que llene las necesidades diarias del animal en crecimiento (Stanton et al. 2012, Krpálková et al. 2014).

Inapropiadas prácticas sanitarias del manejo de terneras, han reportado problemas gastrointestinales o enfermedad respiratoria durante el periodo predestete, que sometidas a algún tratamiento, presentan menor ganancia de peso diario, lo cual conlleva a un valor de EPP elevado (de Passillé y Rushen 2016, Heinrichs et al. 2005)and were allocated to 3 weaning strategies: $(1$; similar a lo obtenido en este estudio con la relación existente entre las prácticas de manejo deficientes y el incremento de la EPP.

Quince de las fincas analizadas reportaron la ejecución de tratamientos tempranos de los animales enfermos, lo cual garantiza que la ganancia de peso diario y el peso final de la ternera al destete se vea menos comprometido. Van Der Fels-Klerx et al. (2002) reportaron que la aparición del síndrome respiratorio bovino, o diarreas en las primeras etapas, no solo llegan a afectar el peso vivo del animal e incrementar la EPP, sino que también contribuyen a incrementar, hasta en un 5\%, la posibilidad de muerte embrionaria temprana o aborto en su primer parto.

Uno de los puntos críticos de la crianza es el manejo del período de calostro, pues se ha reportado que un $40 \%$ de las muertes pre-destete, asociados a un fallo en la transferencia de la inmunidad pasiva, lo cual se obtiene en la ingesta de calostro (Hulbert y Moisá 2016). Los animales de baja vitalidad producto de fallas de manejo como falta de higiene o ambientes hostiles, necesitan terapias con antinflamatorios para mejorar la capacidad de absorción de inmunoglobulinas, pues la respuesta inflamatoria inhibe la transferencia de inmunidad pasiva del calostro, a pesar de tener una ingesta constante (Hulbert y Moisá
2016). Las fincas analizadas ofrecen el calostro en las primeras horas y la mayoría de fincas utiliza leche entera para alimentar las terneras de buena calidad. Todos reportan al menos, una muerte o signos de enfermedad en las crías los primeros meses de vida.

De las fincas analizadas, 5 utilizan reemplazador de leche para alimentar las terneras durante su período de lactancia, 16 utilizan leche entera y solo una reporta usar ambos tipos. Solo 8 de las fincas realizaban análisis previo a la leche, principalmente para controlar residuos de antibióticos y mastitis. La administración de leche entera sin ningún tipo de análisis previo o pasteurización, provocó la incidencia de diarreas y disminuye la tasa de crecimiento de la ternera. Hubo casos de leche con altos niveles de células somáticas, mastitis o residuos de antibiótico que afectan de forma directa la salud de la hembra (Edrington et al. 2012). Existen reportes que asocian el consumo de leche de descarte a un incremento de la EPP, a diferencia de aquellas que consumieron leche de buena calidad (Mohd Nor et al. 2013).

Aparte de la calidad de la leche, los ingredientes utilizados en las dietas de terneras lactantes son importantes para garantizar el desarrollo ruminal adecuado. La totalidad de fincas estudiadas ofrece concentrado y heno o pasto verde, con la finalidad de incrementar la producción de ácidos grasos volátiles, principalmente butirato que estimula el crecimiento de las papilas ruminales y mejora la función del rumen (Khan et al. 2011).

El desarrollo adecuado de una ternera se ve perjudicado de forma directa por el alojamiento, inadecuada infraestructura que puede afectar el acceso al agua, alimento y áreas de descanso, al incrementar la desigualdad de los animales y afecta el desarrollo de aquellos que no se encuentran en una buena posición social en la jerarquía del grupo (Lievaart et al. 2012). Del mismo modo, estas carencias van a influir en la incidencia de enfermedades gastrointestinales y respiratorias que a la larga, retrasan la EPP (Khan et al. 2011). Pettersson et al. (2001), reportan mayor cantidad de neumonías y brotes de diarrea, 
cuando se alojan las terneras de forma grupal durante sus primeros meses de vida; asociado al tipo de medidas higiénicas utilizadas en este sistema, el incremento de los niveles de amonio, alta humedad relativa y pobre ventilación.

Fallas en el alojamiento, como poco espacio en el comedero, promueve la competencia que modifica el comportamiento alimenticio de la ternera, e interfiere con la ganancia de peso diario (Lievaart et al. 2012). De la misma manera, el clima al cual se someten las terneras durante sus primeros días, afecta el estado de salud, pues animales bajo estrés calórico disminuyen el consumo de alimentos; así, es necesario tomar medidas para disminuir la temperatura ambiental y garantizar el incremento de consumo de materia seca que se pierde con las altas temperaturas (Lievaart et al. 2012). De las fincas seleccionadas, 14 mantienen las terneras en alojamiento individual. La mayoría cuenta con piso elevado o camas de tipo orgánico; lo que permite saber, con certeza, el consumo de alimento sólido para obtener el peso adecuado al momento del destete (Wathes et al. 2008).

Al analizar el efecto del manejo, en asociación con la raza, se puede observar una tendencia al incremento en la EPP conforme las prácticas pierden eficacia (Figura 2). Esta tendencia es más marcada en las vacas Jersey. Los animales desarrollados en sistemas de crianza de alta calidad presentan una EPP de 26,1 (IC95\%: 25,6-26,7), lo cual difiere significativamente, de las terneras criadas en sistemas de media y baja calidad, que tuvieron una EPP de 29,4 (IC95\%: 29,0229,8) y 29,9 (IC95\%: 29,3-30,5), respectivamente. Por otra parte, en la raza Holstein, no se observaron diferencias importantes entre las terneras criadas bajo sistemas de calidad media (EPP: 30,7; IC95\%: 30,2-31,2) o baja (EPP 30,5, IC95\%: 30,1-30,8), pero sí se observó que las vacas desarrolladas, en sistemas de crianza con manejo de alta eficiencia, presentaron las EPP más bajas (EPP: 29,2, IC95\%: 28,6-29,8) (Figura 2).

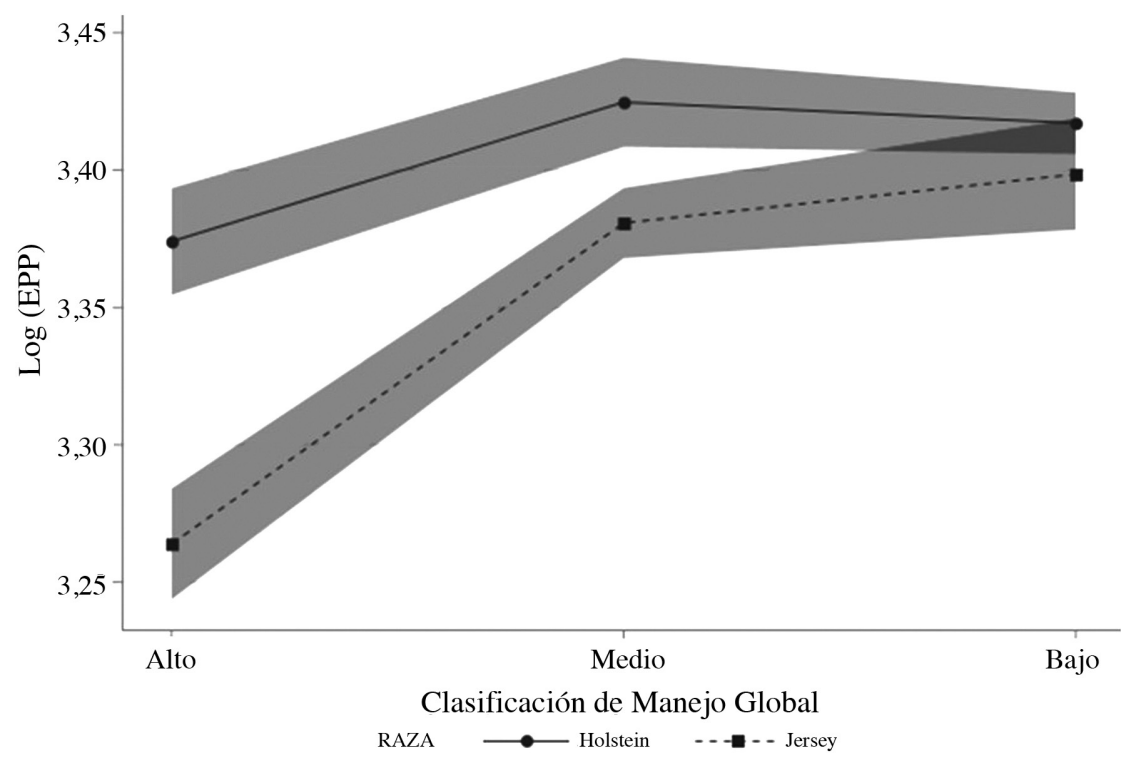

Fig. 2. Medias marginales e intervalo de confianza 95\% para Edad a Primer Parto (EPP, escala logarítmica) según raza (Jersey y Holstein) y categoría de manejo global (alto, medio y bajo). 
Los valores anteriores demuestran que cuando se analiza el efecto del manejo (variable modificable) sobre la EPP, las fincas con manejo medio presentan resultados que se asemejan más a las fincas de manejo deficiente, en comparación con aquellas con alta eficiencia en su método de crianza. Esto se puede deber a que dentro de las categorías de la variable de manejo global, existen aspectos que pesan más sobre la EPP. Por ejemplo, una mayor incidencia de enfermedades gastrointestinales y respiratorias, durante el desarrollo temprano de una ternera, puede provocar un incremento mayor en la EPP que la periodicidad de visita del médico veterinario a la finca. Por tanto, las diferencias entre las fincas, con alto y medio manejo, se marcan más sobre el efecto de la EPP. Asimismo, vuelve a quedar en evidencia que, independientemente de la raza que se utilice, el tipo de crianza influye de forma directa sobre el valor de la EPP.
Los resultados muestran que el desarrollo de la ternera, bajo condiciones adecuadas de nutrición, salud y ambiente, mejoran el peso corporal y permiten alcanzar la madurez sexual a edades más tempranas; lo cual, a largo plazo, se puede manifestar en mejores índices productivos y reproductivos dentro del hato (Le Cozler et al. 2008).

No obstante, se pudo observar que existe una alta variación entre las EPP de diferentes hatos, aun cuando sean pertenecientes a un mismo nivel de manejo, tanto para la raza Holstein (Figura 3) como para la Jersey (Figura 4), lo que en muchos casos se puede asociar a la política reproductiva de cada finca; asimismo, el estudio no consideró las posibles diferencias en el manejo posdestete, lo cual puede contribuir a que se observen estas diferencias marcadas en fincas que tienen similar manejo predestete.

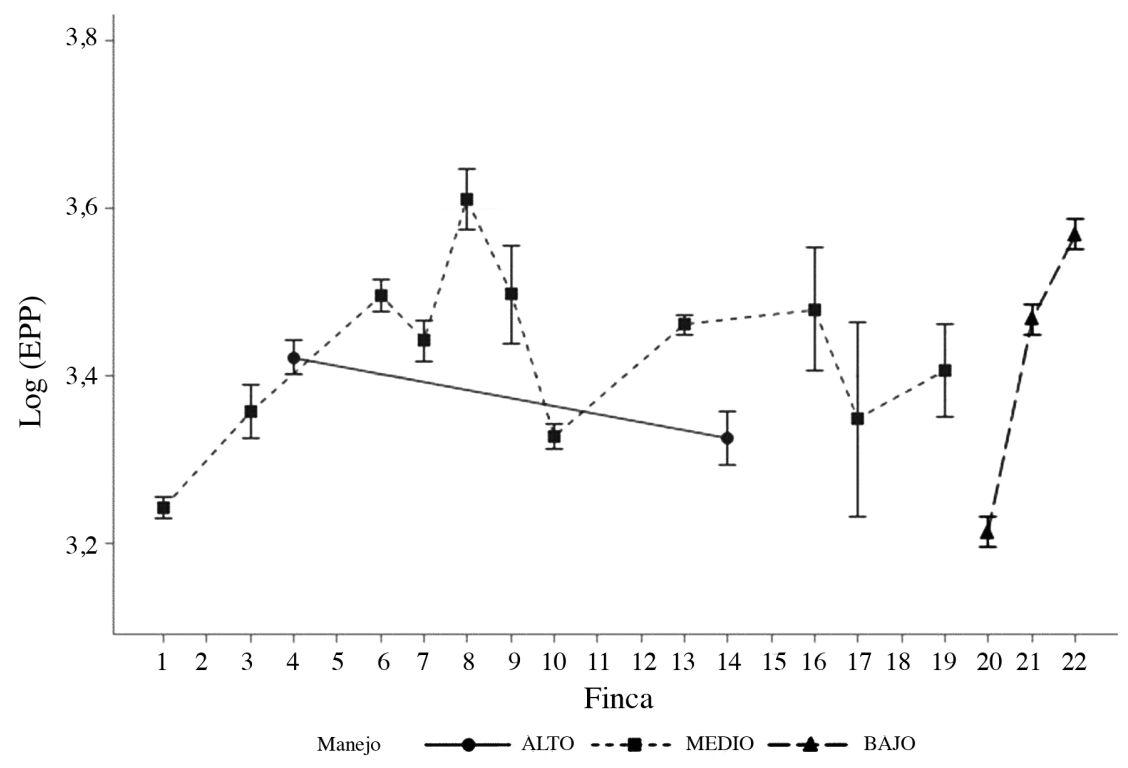

Fig. 3. Medias marginales e intervalos de confianza 95\% para la variable Edad a Primer Parto (EPP, escala logarítmica) en fincas con raza Holstein agrupadas según nivel de manejo (alto, medio, bajo). 


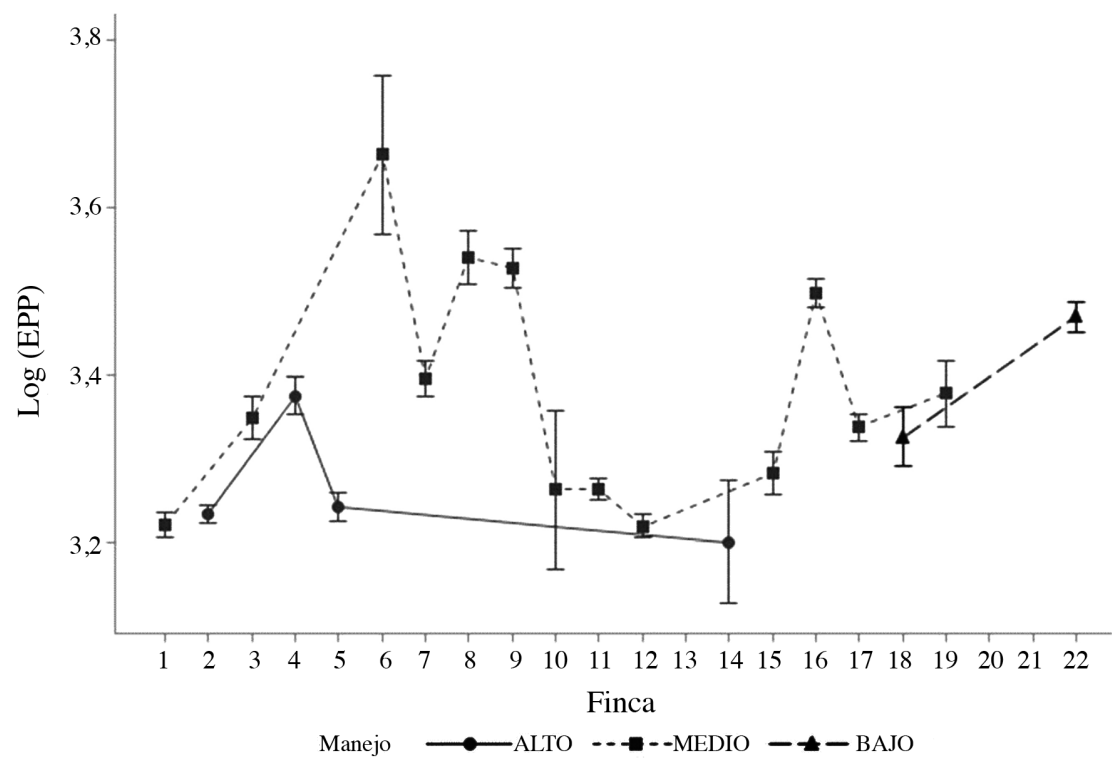

Fig. 4. Medias marginales e intervalos de confianza 95\% para la variable Edad a Primer Parto (EPP, escala logarítmica) en fincas con raza Jersey agrupadas según nivel de manejo (alto, medio, bajo).

Para la raza Holstein, las fincas que presentaron valores de EPP mayores, corresponden a las Fincas 8 (EPP: 37,0; IC95\%: 35,7-38,4) y 22 (EPP: 35,5; IC95\%: 34,9-36,1), las cuales tienen hatos ubicados en Región Huetar Norte (Figura 3). La Finca 8 tiene un tipo de manejo medio y la 20 un manejo de baja calidad. Por otra parte, los valores de EPP más bajos se reportan en las fincas 1 (EPP: 25,6; IC95\%: 25,3-26,0), correspondiente a una de las fincas con manejo de alta calidad y la 20 (EPP: 24,9; IC95\%: 24,5-25,4), que se encuentra en los hatos de técnicas de crianza de baja calidad.

Como caso particular, en la Finca 20, las terneras se alojan de forma grupal los primeros 60 días de vida. La finca mantiene una política de reemplazos abierta, en la cual se crían animales dentro del hato, aunque también se adquieren hembras fuera de la finca; tampoco hay análisis de la leche entera que se utiliza como fuente de alimentación de las terneras lactantes. Estas características se podrían considerar como posibles factores de riesgo a incrementar la EPP. Sin embargo, a pesar de todo esto, la finca mantiene un valor bajo de EPP, posiblemente asociado a que llevan sus animales a primer servicio a la temprana edad de 12 meses; además, hacen uso del toro si las novillas repiten celo.

La mayoría de las fincas, con ganado raza Jersey, presentan valores inferiores de EPP en relación con las fincas con ganado Holstein (Figura 4). La Finca 6 presenta el valor más alto de EPP reportado, a pesar de tener un buen manejo de crianza (EPP: 39,0; IC95\%: 35,5-42,9). Los valores de EPP inferiores que reporta la raza Jersey concuerdan con lo reportado en estudios anteriores, que manifiestan la precocidad de la raza para alcanzar su madurez reproductiva (Castillo-Badilla et al. 2013).

Al analizar el efecto de la finca y las prácticas de manejo, sobre la edad del inicio de la vida reproductiva, se refleja la diversidad que existe, actualmente, en los sistemas de producción costarricenses, en la cual se encuentra gran variación en aspectos tales como sistemas 
de confinamiento, tipos raciales, nivel de uso de tecnologías, manejo de recurso forrajero, uso de suplementos y concentrados, mano de obra, entre otros (Vargas Leitón et al. 2013).

Dentro del grupo de las fincas con superior calidad de manejo, corresponden en su mayoría a explotaciones especializadas de altura, ubicadas en la zona de Vara Blanca. Según Vargas Leitón et al. (2013), estas fincas se caracterizan por ser lecherías intensivas, altamente tecnificadas, generalmente establecidas sobre los $1600 \mathrm{msnm}$, con ganado puro, especializado y con la mayor producción de sólidos por hectárea. Asimismo, ofrecen dietas de alta calidad, utilizan concentrado, suplementos y fertilizantes en los pastos. Esto puede explicar la presencia de una EPP entre los 24-28 meses de edad. Sin embargo, la finca 6, incluida en este grupo, reporta una EPP de 37,9 meses, lo cual se puede deber a que es la única finca, dentro de este grupo, localizada en bajura, en una zona que presenta temperatura ambiental y humedad relativa superior a las demás. Estos factores climáticos hacen más difícil el inicio de la vida reproductiva a una edad más temprana, debido a los pastos de baja calidad que crecen en la zona y al estrés calórico al que se someten los animales (Wing-Ching Jones et al. 2008).

El grupo de fincas con tipo de manejo medio es el más diverso, en el cual tanto la EPP, como la ubicación de las fincas, es variable. Por otra parte, las explotaciones con manejo de baja calidad son, en su mayoría, fincas ubicadas en la zona de bajura costarricense, principalmente en la región Huetar Norte, en donde las temperaturas son altas y las precipitaciones superiores a los $4000 \mathrm{~mm}$ anuales, e igualmente, se tiene una mayor incidencia de sistemas de pastoreo continuo (Vargas Leitón et al. 2013). Esto se puede relacionar, entre otros factores, al índice de temperatura y humedad (ITH), pues, al aumentar la precipitación, la temperatura baja y la humedad relativa aumenta, con una relación superior a 72 en el ITH; el animal se somete a un estado de estrés calórico que afecta, de forma directa, el desempeño reproductivo; por tanto a un incremento de la EPP (Wing-Ching Jones et al. 2008).

\section{CONCLUSIONES}

Los resultados demuestran la importancia del efecto de los factores modificables sobre la EPP, lo cual evidencia que las prácticas de manejo predestete influyen, directamente, sobre el valor de la EPP, lo cual se convierte en una herramienta de utilidad para los productores, ya que les permite conocer qué aspectos del desarrollo de sus reemplazos, pueden mejorar para obtener beneficios a corto, mediano y largo plazo, en especial cuando se identifican las variables modificables existentes en la finca. Se puede concluir que los rubros de alimentación y alojamiento son los que se acercan más al puntaje máximo por categoría, lo cual sugiere la probabilidad de que las fincas fallen más en el manejo sanitario de las terneras que en el alojamiento y alimentación. Errores comunes cometidos dentro de los protocolos sanitarios, de alimentación y alojamiento, pueden ser corregidos de forma temprana para evitar un impacto negativo sobre los parámetros reproductivos de una novilla e incrementar la eficiencia del hato en general.

\section{LITERATURA CITADA}

Abeni, F; Calamari, L; Stefanini, L; Pirlo, G. 2012. Effect of average daily gain on body size, metabolism, and milk production of Italian Holstein heifers raised on two different planes of nutrition and calving at two different ages. Livestock Science 149(1-2):7-17.

Baaijen, M; Pérez, E. 1995. Information technology in the Costa Rican dairy sector: A key instrument in extension and on-farm research. Agric. Hum.Values 2:45-51.

Bolker, BM; Brooks, ME; Clark, CJ; Geange, SW; Poulsen, JR; Stevens, MHH; White, JSS. 2009. Generalized linear mixed models: a practical guide for ecology and evolution. Trends in Ecology and Evolution 24(3):127-135.

Brand, A; Noordhuizen, JPTM; Schukken, YH. 1997. Herd Health and Production Management in dairy practice. segunda ed. s.1.,The Netherlands, Wageningen Pers. 543 p.

Castillo Badilla, G; Salazar Carranza, M; Murillo Herrera, J; Romero Zúñiga, JJ. 2013. Factores que afectan la edad al primer parto en vacas Jersey de lechería especializada en Costa Rica. Revista Ciencias Veterinarias 29(1):7-19. 
Castillo Badilla, G; Vargas Leitón, B; Hueckmann Voss, F; Romero Zúñiga, JJ. 2019. Factores que afectan la producción en primera lactancia de vacas lecheras de Costa Rica. Agronomía Mesoamericana 30:209-227.

Changhee Do; Nidarshani, W; Kwanghyun, C; Yunho, C; Taejeong, C; Byungho, P; Donghee, L. 2013. The effect of age at first calving and calving interval on productive life and lifetime profit in Korean Holsteins. Australasian Journal of Animal Sciences 26(11):1511-1517.

Cooke, JS; Cheng, Z; Bourne, NE; Wathes, DC. 2013. Association between growth rates, age at first calving and subsequent fertility, milk production and survival in Holstein-Friesian heifers. Open Journal of Animal Sciences 3(1):1-12.

de Passillé, AM; Rushen, J. 2016. Using automated feeders to wean calves fed large amounts of milk according to their ability to eat solid feed. Journal of Dairy Science 99(5):3578-3583.

Dwinger, R.; Capella, E; Perez, E; Baaijen, M. 1994. Application of a computarized herd management and production control program in Costa Rica. Trop Agric v. 71(1):74-76.

Edrington, T; Dowd, S; Farrow, R; Hagevoort, G; Callaway, T; Anderson, R; Nisbet, D. 2012. Development of colonic microflora as assessed by pyrosequencing in dairy calves fed waste milk Journal of Dairy Science 95:4519-4525.

Ettema, JF; Santos, JEP. 2004. Impact of age at calving on lactation, reproduction, health, and income in firstparity Holsteins on commercial farms. Journal of Dairy Science 87(8):2730-2742.

Hare, E; Norman, HD; Wright, JR. 2006. Trends in calving ages and calving intervals for dairy cattle breeds in the United States. Journal of Dairy Science 89:365-370.

Heinrichs, AJ; Heinrichs, BS. 2011. A prospective study of calf factors affecting first-lactation and lifetime milk production and age of cows when removed from the herd. Journal of Dairy Science 94(1):336-341.

Heinrichs, AJ; Heinrichs, BS; Harel, O; Rogers, GW; Place, NT. 2005. A Prospective study of calf factors affecting age, body size, and body condition score at first calving of holstein dairy heifers. Journal of Dairy Science 88(8):2828-2835.

Hulbert, LE; Moisá, SJ. 2016. Stress, immunity, and the management of calves. Journal of Dairy Science 99(4):3199-3216.

Hultgren, J; Svensson, C. Pehrsson, M. 2011. Rearing conditions and lifetime milk revenues in Swedish dairy cows. Livestock Science 137:108-115.

Ibekwe, R. 2015. Modifiable risk factors of hypertension and socio-demographic profile in Oghara, Delta State; prevalence and correlates. (en línea). Annals of medical and health sciences research 5(1):71-77. DOI: https://doi.org/10,4103/2141-9248,149793.
Khan, MA; Weary, DM; Von Keyserlingk, MAG. 2011. Invited review: Effects of milk ration on solid feed intake, weaning, and performance in dairy heifers. Journal of Dairy Science 94:1071-1081.

Krpálková, L; Cabrera, VE; Kvapilík, J; Burdych, J; Crump, P. 2014. Associations between age at first calving, rearing average daily weight gain, herd milk yield and dairy herd production, reproduction, and profitability. Journal of Dairy Science (97):1-10.

Le Cozler, Y; Lollivier, V; Lacasse, P; Disenhaus, C. 2008. Rearing strategy and optimizing first-calving targets in dairy heifers: a review. Animal 2(9):1393-1404.

Lievaart, J; Heinrichs, J; Boersema, J; Mee, J. 2012. Rearing heifers for optimal growth and future performance. In XXVII World Buiatrics Congress. Lisbon, Portugal, sp.

Meale, S; Chaucheyras-Durand, F; Berends, H; Guan, LL; Steele, MA. 2017. From pre to postweaning: Transformation of the young calfs gastrointestinal tract. Journal of Dairy Science 100:1-12.

Mee, JF; Sánchez-Miguel, C; Doherty, M. 2014. Influence of modifiable risk factors on the incidence of stillbirth/ perinatal mortality in dairy cattle. The Veterinary Journal 199(1):19-23.

Mohd Nor, N; Steeneveld, W; van Werven, T; Mourits, MCM; Hogeveen, H. 2013. First-calving age and first-lactation milk production on Dutch dairy farms. Journal of Dairy Science 96(2):981-992.

Nilforooshan, MA; Edriss, MA. 2004. Effect of age at first calving on some productive and lLongevity traits in Iranian Holsteins of the Isfahan Province. Journal of Dairy Science 87:2130-2135.

Noordhuizen, JP; Buurman, J. 1984. VAMPP: a veterinary automated management and production control programme for dairy farms (the application of MUMPS for data processing). The Veterinary Quarterly 6(2):66-72.

Ossa, GA; Suárez, MA; Pérez, JE. 2007. Factores ambientales y genéticos que influyen la edad al primer parto y el intervalo entre partos en hembras de la raza criolla Romosinuano. Revista Corpoica Ciencia y Tecnología Agropecuaria 8(2):74-80.

Pérez, E; Baaijen, M; Capella, E; Barkema, HW. 1989. Development of a livestock information system for Costa Rica. In 6th International Conference of Institutes for Tropical Veterinary Medicine. Utrecht, The Netherlands, s.e. p. 221-224.

Pettersson, K; Svensson, C; Liberg, P. 2001. Housing, feeding and management of calves and Replacement heifers in Swedish Dairy Herds. Acta Vet. Scand 42(4):465-478.

Pirlo, G; Miglior, F; Speroni, M. 2000. Effect of age at first calving on production traits and on difference between milk yield returns and rearing costs in Italian Holsteins. Journal of Dairy Science 83(3):603-608. 
Pithua, P; Wells, SJ; Godden, SM; Raizman, EA. 2009. Clinical trial on type of calving pen and the risk of disease in Holstein calves during the first $90 \mathrm{~d}$ of life. Preventive Veterinary Medicine 89:8-15.

Roche, JR; Dennis, NA; MacDonald, KA; Phyn, CVC; Amer, PR; White, RR; Drackley, JK. 2015. Growth targets and rearing strategies for replacement heifers in pasture-based systems: A review. Animal Production Science 55(7):902-915.

Salazar-Carranza, M; Castillo-Badilla, G; Murillo-Herrera, J; Hueckmann-Voss, F; Romero-Zúñiga, JJ. 2013. Edad al primer parto en vacas Holstein de lechería especializada en Costa Rica. Agronomía Mesoamericana 24(2):233-243.

SAS Institute Inc (Statistical Analysis Software). 2010. SAS/ STAT 9,22 User's Guide.

Stanton, L; Kelton, DF; Leblanc, SJ; Wormuth, J; Leslie, K. 2012. The effect of respiratory disease and a preventative antibiotic treatment on growth, survival, age at first calving, and milk production of dairy heifers. Journal of Dairy Science 57:4950-4960.

Van Der Fels-Klerx, HJ; Saatkamp, HW; Verhoeff, J; Dijkhuizen, AA. 2002. Effects of bovine respiratory disease on the productivity of dairy heifers quantified by experts. Livestock Production Science 75:157-166.

Vargas Leitón, B; Solís Guzmán, O; Saenz Segura, F; León Higaldo, H. 2013. Caracterización y clasificación de hatos lecheros en Costa Rica mediante análisis multivariado. Agronomía Mesoamericana 24(2):257-275.

Vargas-Leitón, B; Ulloa, J. 2008. Relación entre curvas de crecimiento y parámetros reproductivos en grupos raciales lecheros de distintas zonas agroecológicas de Costa Rica. Livestock Research for Rural Development 20(7):1-17.

Washburn, SP; Silvia, WJ; Brown, CH; Mcdaniel, BT; Mcallister, AJ. 2002. Trends in reproductive performance in Southeastern Holstein and Jersey DHI herds. Journal of Dairy Science 85:244-251.

Wathes, DC; Brickell, JS; Bourne, NE; Swali, A; Cheng, Z. 2008. Factors influencing heifer survival and fertility on commercial dairy farms. Animal The Animal Consortium 2(8):1135-1143.

Wing-Ching Jones, R; Pérez, R; Salazar, E. 2008. Condiciones ambientales y producción de leche de un hato de ganado Jersey en el trópico húmedo: El caso del módulo lechero SDA/UCR. Agronomía Costarricense 32(1):87-94. 